\title{
Morfologia bucal interna dos girinos de Leptodactylus labyrinthicus Spix, 1824 (Amphibia: Anura: Leptodactylidae)
}

\author{
Núbia Miranda ${ }^{1}$; Adelina Ferreira ${ }^{1,2}$ \\ ${ }^{1}$ Departamento de Biologia e Zoologia, Instituto de Biociências, \\ Universidade Federal de Mato Grosso - UFMT, \\ Av. Fernando Correa da Costa, s/n, Coxipó, CEP 78060-900, Cuiabá, MT, Brasil \\ ${ }^{2}$ Autor para correspondência: Adelina Ferreira, e-mail: adelina@cpd.ufmt.br
}

Miranda, N.; Ferreira, A. Internal buccal morphology of the tadpoles of Leptodactylus labyrinthicus Spix, 1824 (Amphibia: Anura: Leptodactylidae). Biota Neotrop., vol. 8, no. 1, Jan./Mar. 2008. Available from: <http:// www.biotaneotropica.org.br/v8n1/pt/abstract?short-communication+bn00908012008>.

\begin{abstract}
The microanatomy of the oral disc and the buccal cavity of the tadpole of Leptodactylus labyrinthicus is described and compared with other species included in the L. pentadactylus species group. Thirty-five tadpoles between stages 25 and 46 had been dissected and analyzed in scanning electron microscopy. The oral and internal buccal morphology was analyzed in tadpoles between stages 36 and 39 . The buccal morphology of the tadpole of L. labyrinthicus is similar to other tadpoles with adaptation to macrophagy, such as the strong queratinization of the jaw, reduction of the internal buccal structures and presence of one glote uncovered, propitious to carnivorous diets. The tadpole of L. pentadactylus species group share this trend to the reduction of the structures of selection and capture of particle, including reduction in the number of the buccal roof and floor arena papillae, reduction or absence of anterior papillae on the buccal pocket, only two postnarial papillae, small lateral ridge papillae and glandular zones restricted in narrow bands on the edge of velum. In the tadpoles of genera Leptodactylus the larval morphology reflects the current groups considered for this genera.
\end{abstract}

Keywords: internal oral morphology, macrophagy, carnivory, Leptodactylus pentadactylus group.

Miranda, N.; Ferreira, A. Morfologia bucal interna dos girinos de Leptodactylus labyrinthicus Spix, 1824 (Amphibia: Anura: Leptodactylidae). Biota Neotrop., vol. 8, no. 1, jan/mar, 2008. Disponível em: <http:// www.biotaneotropica.org.br/v8n1/pt/abstract?short-communication+bn00908012008>.

Resumo: A microanatomia do disco oral e da cavidade bucal da larva de Leptodactylus labyrinthicus é descrita e comparada com outras espécies do gênero pertencentes ao grupo de $L$. pentadactylus. Trinta e cinco girinos entre os estágios 25 e 46 foram dissecados e analisados em microscopia eletrônica de varredura. A descrição da morfologia oral foi feita utilizando larvas entre os estágios 36 e 39. A morfologia oral da larva de L. labyrinthicus é similar às descritas para outros girinos com adaptação a macrofagia, com forte queratinização das coberturas das mandíbulas, redução das estruturas bucais internas e presença de uma glote descoberta, propícias às dietas carnívoras. As larvas do grupo de L. pentadactylus compartilham essa tendência à redução das estruturas de seleção e captura de partículas, apresentando redução no número de papilas da arena do assoalho e do teto bucal, redução ou ausência das papilas anteriores às bolsas bucais, apenas duas papilas pós-nasais, pequenas papilas laterais à crista mediana e zonas glandulares restritas a estreitas faixas no bordo velar. Nos girinos do gênero Leptodactylus a morfologia larvária reflete os grupos atuais propostos para o gênero.

Palavras-chave: morfologia oral interna, macrofagia, carnivoria, grupo L. pentadactylus. 


\section{Introdução}

Estudos relacionados com a microanatomia oral de larvas de anuros têm provido informações acerca de adaptações morfológicas aos mais variados tipos de hábitats e auxiliado na compreensão das relações taxonômicas da Ordem Anura. Os caracteres larvais são muito utilizados como ferramentas complementares nos estudos das relações ecológicas e filogenéticas dos anuros (Wassersug 1976, Wassersug \& Heyer 1988, Spirandeli-Cruz 1991, d'Heursel \& de Sá 1999, Echeverría \& Lavilla 2000, Eterovick \& Sazima 2000, Haas 2003, Pugener et al. 2003, Weber \& Carasmaschi 2006).

O gênero Leptodactylus Fitzinger 1826 abrange 81 espécies que ocorrem na América do Norte, América Central e América do Sul (Frost 2007). Heyer (1969) propôs cinco grupos de espécies para o gênero, baseado em características morfológicas, ecológicas e comportamentais. Os grupos L. melanonotus e L. ocellatus apresentam características que os classificam como sendo grupos mais basais, seguidos pelo grupo $L$. pentadactylus e finalmente pelos grupos L. fuscus e L. marmoratus, tidos como os mais derivados (Heyer 1969). De modo geral, as espécies pertencentes a este gênero apresentam pouca diversidade morfológica quando adultos, sendo assim, as características dos girinos podem ser informativas e, portanto, podem auxiliar na compreensão das relações sistemáticas do grupo (Heyer 1979).

Dezenove espécies pertencem ao grupo de Leptodactylus pentadactylus (Heyer 1969), das quais somente doze possuem a larva descrita: Leptodactylus fallax (Davies et al. 2000), L. flavopictus (Bokermann 1957), L. knudseni (Hero 1990), L. labyrinthicus (Vizotto 1967), L. lithonaetes (Heyer 1995), L. pentadactylus (Hero 1990, Savage 2002) L. rhodonotus (Heyer 1979), L. rhodomystax (Hero 1990, Rodrigues et al. 2007), L. rugosus (Heyer \& Thompson 2000), L. savagei (Heyer 1970) e L. syphax (Eterovick \& Sazima 2000), L. vastus (Vieira et al. 2007) e somente três têm a morfologia bucal interna conhecida: Leptodactylus knudseni (Wassersug \& Heyer 1988), L. pentadactylus (Wassersug \& Heyer 1988) e L. vastus (Vieira et al. 2007).

Leptodactylus labyrinthicus (Spix 1824) ocorre nas regiões de Cerrado e Caatinga do Brasil, além do Paraguai e algumas regiões da Bolívia (Frost 2007). É uma espécie de grande porte, que deposita ovos em ninhos de espuma em bacias escavadas nas margens dos corpos d'água (Agostinho et al. 2002, França et al. 2004, Silva et al. 2005, Zina \& Haddad 2005). Neste trabalho descrevemos a microanatomia do disco oral e da cavidade bucal da larva de Leptodactylus labyrinthicus e comparamos com as de outras espécies do gênero Leptodactylus pertencentes ao grupo de L. pentadactylus.

\section{Material e Métodos}

A desova de Leptodactylus labyrinthicus foi coletada próxima ao Córrego Monjolinho ( $15^{\circ} 36^{\prime} \mathrm{S}$ e $\left.56^{\circ} 03^{\prime} \mathrm{W}\right)$ na Fazenda Buriti, no município de Chapada dos Guimarães, Mato Grosso, Brasil, em dezembro de 2004 e mantida em recipientes plásticos contendo água de nascente, por trinta e sete dias. A água era substituída gradualmente a cada três dias. Foi oferecida diariamente, aproximadamente $25 \mathrm{~g}$ de ração para gato macerada como recurso alimentar alternativo. Girinos em diferentes estágios foram fixados periodicamente em paraformaldeído $4 \%$ e conservados em álcool $70 \%$. Os estágios de desenvolvimento foram determinados de acordo com Gosner (1960). Trinta e cinco girinos entre os estágios 25 e 46 foram dissecados e analisados em Microscopia Eletrônica de Varredura (MEV). A descrição da morfologia oral foi feita utilizando larvas entre os estágios 36 e 39. A caracterização morfológica e a terminologia adotada foram baseadas em Wassersug (1976) e Altig \& McDiarmid (1999). Trinta girinos de Leptodactylus labyrinthicus entre os estágios 25 e 45 estão depositados na Coleção Zoológica, do Instituto de Biociências da Universidade Federal de Mato Grosso, Brasil (lotes UFMT 4747, 4748 e 4749).

\section{Microscopia Eletrônica de Varredura}

Antes de iniciar a dissecação cada larva foi lavada em água e mergulhada em solução de azul de metileno a $1 \%$, para facilitar a visualização das estruturas orais. Sob microscópio estereoscópico e com tesoura de microdissecção, iniciou-se uma incisão cuidadosa pela comissura labial (direita e esquerda) até se visualizar o limite das coberturas da mandíbula ventral e dorsal. A partir daí o corte seguiu em direção postero-dorsal. A separação total das porções dorsal e ventral foi realizada através de um corte transversal. Seguindo basicamente o procedimento de dissecação descrito por Wassersug (1976). As amostras foram desidratadas em concentrações crescentes de álcool, secas ao ponto crítico e cobertas com ouro paládio em aparelho do tipo sputtering, analisadas e fotodocumentadas através de microscópio eletrônico de varredura (Jeol JSM 5800LV).

\section{Resultados}

\section{Descrição morfológica}

O disco oral da larva de Leptodactylus labyrinthicus é ligeiramente emarginado ventralmente. As papilas marginais grossas e curtas estão dispostas em uma fileira unisseriada alternada, na qual as bases estão dispostas em fileira, mas o encontro dos ápices dá a falsa impressão de uma fileira bisseriada. O lábio anterior apresenta uma ampla interrupção. As coberturas das mandíbulas apresentam serrações cônicas e são fortemente queratinizadas, sendo a superior com formato de arco e a inferior em V. A fórmula dentária é 1/2(1), sendo as fileiras de dentículos córneos P1 e P2 de mesmo comprimento (Figura 1).

O assoalho bucal é triangular (Figura 2a). Quatro papilas infralabiais estão presentes, o par anterior localizado na linha mediana apresenta as bases das papilas fundidas. Estas são cilíndricas e apresentam toda a superfície da parede anterior e o ápice coberto por pústulas. As outras duas papilas são quadrangulares, mais baixas e mais largas (cerca do triplo da largura das papilas do par anterior),

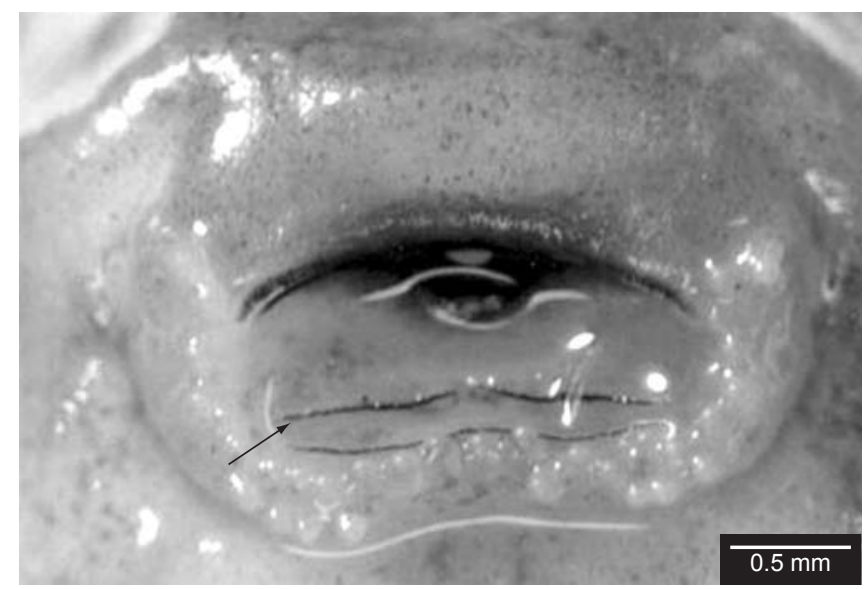

Figura 1. Disco oral da larva de Leptodactylus labyrinthicus no estágio 36. A seta indica a primeira fileira posterior (P1) de dentículos córneos, que aparece fragmentada neste exemplar.

Figure 1. Oral disc of Leptodactylus labyrinthicus tadpole at stage 36. The arrow indicates the first posterior tooth (P1), that appears fragmented in this tadpole. 

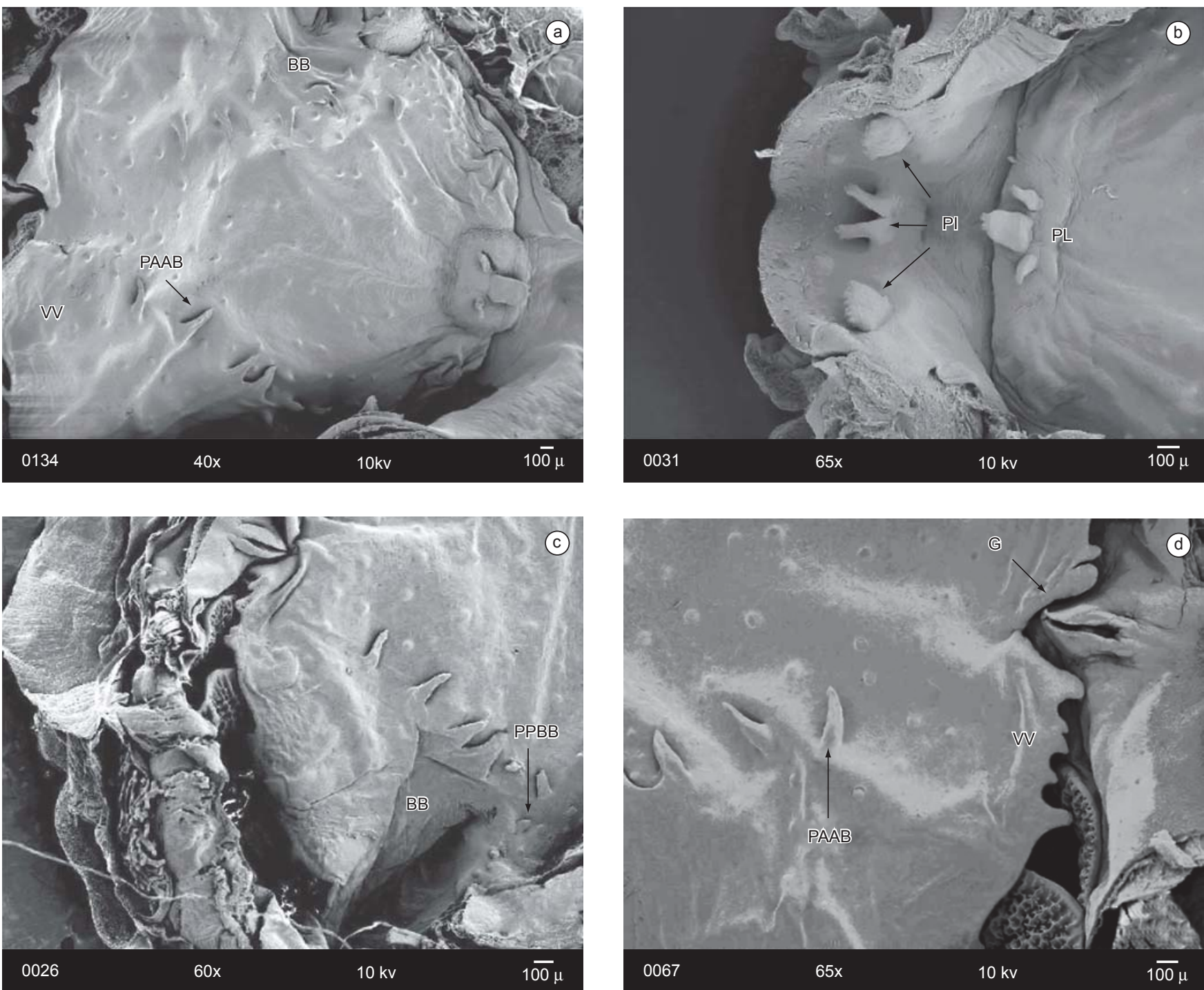

Figura 2. a) Assoalho bucal da larva de Leptodactylus labyrinthicus (estágio 37); b) Papilas infralabiais e linguais (estágio 36); c) Bolsa bucal e velo ventral (estágio 37); e d) Glote e velum ventral (estágio 36). PAAB: papila da arena do assoalho bucal, BB: bolsa bucal, G: glote, PI: papila infralabial, PL: papila lingual, PPBB: papilas pré-bolsa bucal, VV: velum ventral.

Figure 2. a) Leptodactylus labyrinthicus tadpole floor of oral cavity (stage 37); b) Infralabial papillae and lingual papillae (stage 36); c) Buccal pocket and ventral velum (stage 37); and d) Glottis and ventral velum (stage 36). PAAB: buccal floor arena papillae, BB: buccal pocket, G: glottis, PI: infralabial papillae, PL: lingual papillae, PPBB: prepocket papillae, VV: ventral velum.

apresentando a borda superior recoberta de pequenas pústulas (Figura $2 b$ ). O rudimento lingual tem formato de elipse e está localizado medialmente logo após a papila infralabial, apresentando três papilas linguais. As duas papilas linguais laterais são cônicas e em menor tamanho do que a papila central, a qual é larga e comprimida no sentido póstero-anterior (Figura $2 b$ ). A arena do assoalho bucal tem formato de trapézio e é delimitada por seis papilas de cada lado, as quais são cônicas, longas e com os ápices dirigidos para o centro da arena. Aproximadamente cinqüenta pústulas estão distribuídas na arena. As bolsas bucais são profundas, largas e dispostas transversalmente (Figura 2c). Perfurações não são visíveis. A região anterior às bolsas bucais contém de quatro a cinco papilas. O velum ventral apresenta projeções digitiformes na borda, as quais são notadamente mais marcantes próximas à glote. A zona glandular ventral é pouco perceptível restringindo-se à superfície dorsal das projeções do velo. A glote encontra-se descoberta e está ligeiramente inclinada no sentido anteroventral (Figura 2d).
O teto bucal é triangular (Figura 3a). Apresenta uma arena prénasal em formato de trapézio. Em sua porção posterior, à frente das coanas destaca-se uma crista em formato de $\mathrm{V}$ recoberta por pústulas. As coanas (Figuras 3c e 3d) são ovais e estão orientadas transversalmente. A parede anterior da narina é mais baixa que a parede posterior e é coberta por pequenas pústulas. Não apresenta papilas pré-nasais. Duas papilas pós-nasais estão presentes, uma de cada lado, longas, cônicas, recobertas por pústulas, com ápices arredondados, os quais são dirigidos para o centro da arena pré-nasal. A arena pós-nasal é triangular, curta e estreita, sendo delimitada posteriormente por uma crista mediana semicircular, a qual apresenta uma leve bifurcação (Figuras 3b, 3c e 3d). As duas papilas laterais da crista, localizadas uma de cada lado da crista mediana, são baixas, cônicas e com seus ápices voltados para a crista (Figuras $3 \mathrm{c}$ e $3 \mathrm{~d}$ ). A arena do teto bucal tem formato de trapézio e é delimitada por duas a três papilas longas e de formato cônico de cada lado, com os ápices voltados para o centro da arena. Cerca de setenta pústulas encontram-se dispersas 

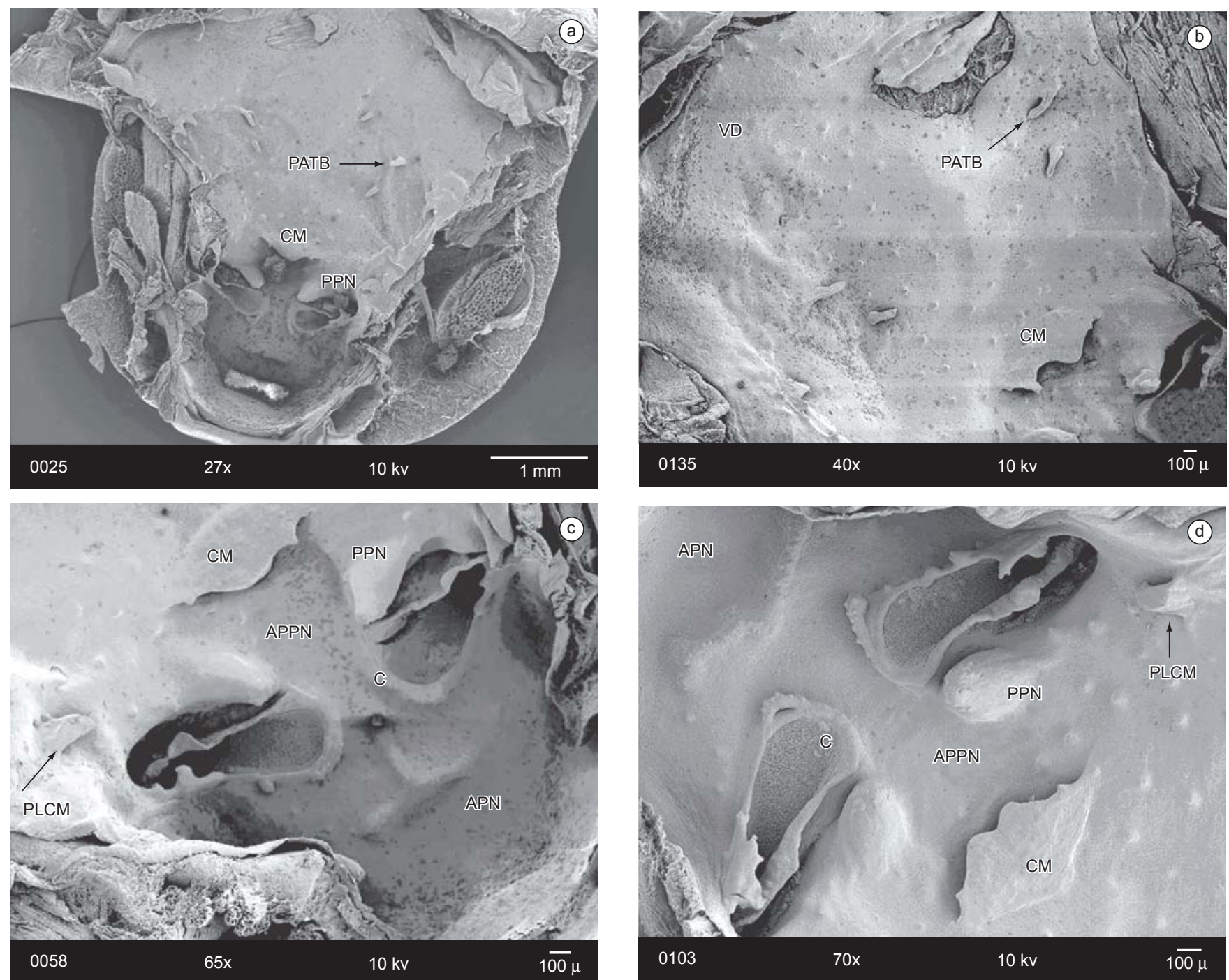

Figura 3. a) Teto bucal da larva de Leptodactylus labyrinthicus (estágio 36); b) Arena do teto bucal (estágio 37); c) Arena pré e pós-nasal (estágio 37); e d) Crista mediana e papilas laterais da crista (estágio 39). APN: arena pré-nasal, APPN: arena pós-nasal, C: coana, CM: crista mediana, PATB: papila da arena do teto bucal, PLCM: papila lateral da crista mediana, PPN: papila pós-nasal, VD: velum dorsal

Figure 3. a) Leptodactylus labyrinthicus tadpole roof of oral cavity (stage 36); b) Buccal roof arena (stage 37); c) Prenarial and postnarial arena (stage 37); and d) Median ridge and lateral ridge papillae (stage 39). APN: prenarial arena, APPN: postnarial arena, C: choana, CM: median ridge, PATB: buccal roof arena papillae, PLCM: lateral ridge papillae, PPN: postnarial papillae, VD: dorsal velum.

no centro da arena. A zona glandular está restrita a uma faixa na porção posterior do velum dorsal, o qual é estreito e não apresenta projeções (Figuras $3 \mathrm{a}$ e $3 b$ ).

\section{Variações nos caracteres da morfologia oral interna}

Alguns caracteres apresentaram variações provavelmente relacionadas ao desenvolvimento ontogenético. Os caracteres variaram na forma, disposição e ornamentação. No assoalho bucal, o par mais externo de papilas infralabiais é menos perceptível nos estágios menos avançados, as papilas não apresentam tantas pústulas na borda superior, mas já apresentam o formato retangular. O par interno já apresenta a conformação descrita para o estágio 36, porém só apresenta três pústulas em cada borda superior, estas pústulas em estágios mais avançados concentram-se na borda anterior das papilas. No teto bucal, a crista presente à frente das coanas apresenta formato de T nos estágios menos avançados e posteriormente adquire o formato de V (Figuras 3c e 3d). A crista mediana apresenta três padrões de bipartição: central e bastante pronunciado conferindo à mesma a forma de $\mathrm{M}$, levemente bipartida no centro em um dos lados da crista (Figura 3b, 3c e 3d). O número de papilas da arena do assoalho e do teto bucal manteve-se inalterado até o estágio 39, a partir do qual começaram a sofrer reduções.

\section{Discussão}

Os cinco grupos propostos para o gênero Leptodactylus (L. marmoratus, L. melanonotus, L. ocellatus, L. pentadactylus e L. fuscus) com base na morfologia e comportamento dos adultos (Heyer 1969), têm sido suportados pelos caracteres bucais internos larvários (Wassersug \& Heyer 1988, Prado \& d'Heursel 2006). Leptodactylus labyrinthicus pertence ao grupo de L. pentadactylus, o qual é constituído por outras espécies que apresentam girinos carnívoros tais como L. flavopictus, L. knudseni e L. rhodomystax (Wassersug \& Heyer 1988, Eterovick \& Sazima 2000, Rodrigues et al. 2007). 
As diferenças na morfologia oral em larvas de anfíbios anuros podem ser interpretadas como diferentes adaptações ao hábitat, ou associadas a distribuição geográfica e ao comportamento alimentar. As adaptações podem ocorrer na morfologia externa (altura das nadadeiras, disposição do disco oral, formato do corpo, etc) ou na morfologia interna, representada pela rede de captação de partículas que é um dos principais papéis das estruturas bucofaríngeas (Wassersug \& Rosenberg 1979, Altig \& McDiarmid 1999, Viertel \& Richter 1999). A morfologia oral aqui descrita para a larva de L. labyrinthicus é similar às descritas para girinos da mesma espécie em outras regiões (Rossa-Feres \& Nomura 2006), e para outros girinos com adaptação a macrofagia (Spirandeli-Cruz 1991), incluindo-se aqui os girinos carnívoros. Estes girinos costumam apresentar forte queratinização das coberturas das mandíbulas e redução das estruturas bucais internas (Altig \& Johnston 1989, Spirandeli-Cruz 1991). Isto provavelmente decorre do hábito carnívoro dos girinos que não necessitam, portanto, de grande número de estruturas bucais para fazer a seleção das partículas alimentares (Spirandeli-Cruz 1991).

A presença de uma glote descoberta é uma característica compartilhada pela maior parte das larvas já descritas pertencentes ao gênero Leptodactylus. Isto, provavelmente se deve aos hábitos alimentares destes girinos: larvas generalistas de hábitos bentônicos (L. ocellatus, Spirandeli-Cruz, 1991), larvas generalistas de ambientes lênticos (grande parte das larvas pertencentes aos grupos L. fuscus e L. melanonotus, Wassersug \& Heyer, 1988, Prado \& d'Heursel, 2006, de Sá et al. 2007) e larvas carnívoras (espécies do grupo L. pentadactylus, Cardoso \& Sazima 1977, Wassersug \& Heyer 1988, Eterovick \& Sazima 2000, Prado et al. 2005, Rodrigues et al. 2007, este estudo). Foi observado, por exemplo, comportamento predatório de larvas de L. labyrinthicus sobre ovos não-fertilizados encontrados no ninho (Agostinho et al. 2002, Prado et al. 2005), sobre ovos em ninhos coespecíficos (Silva et al. 2005) ou sobre larvas de outras espécies (Cardoso \& Sazima 1977, Silva et al. 2005). Como a alimentação consiste na ingestão em sua maioria de partículas relativamente grandes, a presença de uma glote descoberta permite que estas partículas tenham acesso livre à glote e possam facilmente ser ingeridas. Wassersug \& Duellman (1984) também sugeriram que a presença de uma glote descoberta é muito importante para a sobrevivência de girinos em ambientes com baixas concentrações de oxigênio dissolvido, o que se justifica pelo fato dos girinos de L. labyrinthicus iniciarem seu desenvolvimento em ninhos de espuma pouco oxigenados, antes de serem carreados até os grandes corpos d'água onde irão completar seu desenvolvimento.

As estruturas observadas no assoalho e teto bucal da larva de L. labyrinthicus são similares às encontradas em outras espécies de Leptodactylus: formato do assoalho e do teto bucal, disposição das bolsas bucais, papilas pré-nasais e velum ventral e dorsal (Wassersug \& Heyer 1988, Spirandeli-Cruz 1991, Prado \& d'Heursel 2006, de Sá et al. 2007, Vieira et al. 2007). Uma das características que mais difere entre os girinos destes grupos é o número de papilas linguais. Nos girinos do grupo de L. fuscus são encontradas quatro papilas linguais, nas espécies do grupo de L. melanonotus as papilas linguais estão ausentes e, como observado nos girinos de L. labyrinthicus, as larvas pertencentes ao grupo de L. pentadactylus e ao grupo de L. ocellatus apresentam de três a quatro papilas linguais (Wassersug \& Heyer 1988, Vieira et al. 2007).

Somente três larvas (Leptodactylus knudseni, L. pentadactylus e $L$. vastus) pertencentes ao grupo de $L$. pentadactylus possuem sua morfologia oral interna descrita (Wassersug \& Heyer 1988, Vieira et al. 2007). Apesar desse número ser muito pequeno quando comparado ao número de espécies pertencentes ao grupo, alguns caracteres têm se mostrado semelhantes e suportam a presença dessas espécies no grupo de $L$. pentadactylus: o formato triangular do assoalho e do teto bucal, a presença de quatro a cinco papilas infralabiais, três a quatro papilas linguais, no máximo dez papilas limitando, a cada lado, a arena do assoalho bucal, crista em formato de $\mathrm{V}$ presente na arena pré-nasal, ausência de papilas pré-nasais, um par de papilas pós-nasais, um par de papilas laterais à crista mediana, de uma a três papilas limitando a arena do teto bucal e zonas glandulares restritas a estreitas faixas no bordo velar. Assim, a principal característica compartilhada pelos girinos deste grupo está relacionada à redução das estruturas de seleção e captura de partículas (Wassersug \& Heyer 1988, Vieira et al. 2007).

As características bucais internas de L. labyrinthicus aqui descritas suportam os grupos propostos por Heyer (1969). Contudo, a descrição da morfologia larvária, incluindo dados da morfologia bucal interna de outros girinos do gênero Leptodactylus é necessária para auxiliar na elucidação das relações entre estes grupos.

\section{Agradecimentos}

Os autores agradecem ao $\mathrm{CNPq}$ pela concessão da bolsa de iniciação científica à Núbia E.O. Miranda (proc. 114466/2005-0), à UFMT e a FAPEMAT (proc. 0769/2006) pelo suporte financeiro ao projeto. À UNICAMP, especialmente à Prof ${ }^{a}$. Dr ${ }^{\mathrm{a}}$ Heidi Dolder, pela concessão do uso do laboratório para análises em microscopia eletrônica de varredura. Os animais foram coletados com autorização do IBAMA/RAN (proc. 02013.004560/04-51).

\section{Referências Bibliográficas}

AGOSTINHO, C.A., FORESTI, F., LIMA, S.L. \& JIM, J. 2002. Reproduction and population size of Leptodactylus labyrinthicus (Amphibia, Anura, Leptodactylidae). Russ. J. Herpetol. 9(1):15-20.

ALTIG, R. \& JOHNSTON, G.F. 1989. Guilds of anuran larvae: relationships among developmental modes, morphologies and habits. Herpetol. Monogr. 2:81-109.

ALTIG, R. \& MCDIARMID, R.W. 1999. Body Plan: Development and Morphology. In Tadpoles, the Biology of Anuran Larvae (Roy W. McDiarmid \& Ronald Altig, eds.). The University of Chicago Press, Chicago and London, p.24-51.

BOKERMANN, W.C.A. 1957. Notas sobre a biologia de "Leptodactylus flavopictus" Lutz, 1926. Rev. Brasil. Biol. 17:495-500.

CARDOSO, A.J. \& SAZIMA, I. 1977. Batracofagia na fase adulta e larvária da Rã-pimenta, Leptodactylus labyrinthicus (Spix, 1824) - (Anura, Leptodactylidae). Cienc. Cult. 29(10):1130-1132.

DAVIES, S.L., DAVIES, R.B., JAMES, A. \& TALYN, B.C.P. 2000. Reproductive behaviour and larval development of Leptodactylus fallax in Dominica, West Indies. Herpetol. Rev. 31:217-220.

DE SÁ, R.O., LANGONE, J.A. \& SEGALLA, M.V. 2007. The tadpole of Leptodactylus notoaktites Heyer, 1978 (Anura, Leptodactylidae). S. Am. J. Herpetol. 2(1):69-75.

D’HEURSEL, A. \& DE SÁ, R.O. 1999. Comparing the tadpoles of Hyla geographica and Hyla semilineata. J. Herpetol. 33:353-361.

ECHEVERRÍA, D.D. \& LAVILLA, E.O. 2000. Internal oral morphology of tadpoles of Dermatonotus muelleri and Elachistocleis bicolor. J. Herpetol. 34(4):517-523.

ETEROVICK, P.C. \& SAZIMA, I. 2000. Description of the tadpole of Leptodactylus syphax, with a comparison of morphological and ecological characters of tadpoles and adults of the species in the L. pentadactylus group (Leptodactylidae, Anura). Amphibia-Reptilia 21:341-350.

FRANÇA, L.F., FACURE, K.G. \& GIARETTA, A.A. 2004. Trophic and spatial niches of two large-sizes species of Leptodactylus (Anura) in Southeastern Brazil. Stud. Neotrop. Fauna E. 39(3):243-248.

FROST, D.R. 2007. Amphibians Species of the World: an Online Reference. American Museum of Natural History, New York, New York. http:// research.amnh.org/herpetology/amphibia (acessado em 28 de Novembro de 2007). 
GOSNER, K.L. 1960. A simplified table for staging anuran embryos and larvae with notes on identification. Herpetologica 16:183-190.

HAAS, A. 2003. Phylogeny of frogs as inferred from primarily larval characters (Amphibia; Anura). Cladistics 19:23-89

HERO, J.M. 1990. An Illustrated key to tadpoles occurring in the Central Amazon rainforest, Manaus, Amazona, Brazil. Amazoniana 11:201-262.

HEYER, W.R. 1969. The adaptive ecology of the species group of the genus Leptodactylus (Amphibia, Leptodactylidae). Evolution 23:421-428.

HEYER, W.R. 1970. Studies on frogs of the genus Leptodactylus (Amphibia: Leptodactylidae). II. Diagnosis and distribution of the Leptodactylus of the Costa Rica. Rev. Biol. Trop. 16:171-205.

HEYER, W.R. 1979. Systematics of pentadactylus species group of the frog genus Leptodactylus (Amphibia: Leptodactylidae). Smithson. Contr. Zool. 301:1-43.

HEYER, W.R. 1995. South American rocky habitat Leptodactylus (Amphibia: Anura: Leptodactylidae) with description of two new species. Proc. Biol. Soc. Wash. 108:695-716.

HEYER, W.R. \& THOMPSON, A.S. 2000. Leptodactylus rugosus. Catalogue of American Amphibian and Reptile 708:1-5.

PRADO, C.P.A. \& D'HEURSEL, A. 2006. The tadpole of Leptodactylus elenae (Anura: Leptodactylidae), with the description of the internal buccal anatomy. S. Am. J. Herpetol. 1(1):79-86.

PRADO, C.P.A., TOLEDO, L.F., ZINA, J. \& HADDAD, C.F.B. 2005. Trophic eggs in the foam nests of Leptodactylus labyrinthicus (Anura, Leptodactylidae): An experimental approach. Herpetol. J. 15:279-284.

PUGENER, L.A., MAGLIA, A.M. \& TRUEB, L. 2003. Revisiting the contribution of larval characters to an analysis of phylogenetic relationships of basal anurans. Zool. J. Linn. Soc-Lond. 139:129-155.

RODRIGUES, D.J., MENIN, M. \& LIMA, A.P. 2007. Redescription of the tadpole of Leptodactylus rhodomystax (Anura: Leptodactylidae) with natural history notes. Zootaxa 1509:61-67.

ROSSA-FERES, D.C. \& NOMURA, F. 2006. Characterization and taxonomic key for tadpoles (Amphibia: Anura) from the northwestern region of São Paulo State, Brazil. Biota Neotropica 6(1): http://www.biotaneotropica. org.br/v6n1/pt/abstract?identification-key+bn00706012006.

SAVAGE, J.M. 2002. The amphibians and reptiles of Costa Rica: a herpetofauna between two continents, between two seas. The University of Chicago Press, Chicago.
SILVA, W. R. DA, GIARETTA, A.A. \& FACURE, K.G. 2005. On the natural history of the South America pepper frog, Leptodactylus labyrinthicus (Spix, 1824) (Anura: Leptodactylidae). J. Nat. Hist. 39(7):555-566.

SPIRANDELI-CRUZ, E.F. 1991. Estudo comparativo da morfologia oral interna de larvas de anfíbios anuros que ocorrem na região de Botucatu, São Paulo (Amphibia, Anura). Tese de Doutorado, Universidade de São Paulo, São Paulo.

VIEIRA, W.L.S., SANTANA, G.G. \& VIEIRA, K.S. 2007. Description of the tadpole of Leptodactylus vastus (Anura: Leptodactylidae). Zootaxa 1529:61-68.

VIERTEL, B. \& RICHTER, S. 1999. Anatomy: Viscera and Endocrines. In Tadpoles, the Biology of Anuran Larvae (Roy W. McDiarmid \& Ronald Altig, eds.). The University of Chicago Press, Chicago and London, p.92-148.

VIZOTTO, L.D. 1967. Desenvolvimento de anuros da região norte-ocidental do Estado de São Paulo. Faculdade de Filosofia, Ciências e Letras, São José do Rio Preto.

WASSERSUG, R.J. 1976. Oral morphology of anuran larvae: terminology and general description. Occas. Papers Mus. Nat. Hist. Univ. Kansas 48:1-23.

WASSERSUG, R.J. \& DUELLMAN, W.E. 1984. Oral structures and their development in egg-brooding hylid frogs embryos and larvae: evolutionary and ecological implications. J. Morphol. 182:1-37.

WASSERSUG, R. J. \& HEYER, W. R. 1988. A survey of internal oral features of Leptodactyloid larvae (Amphibia: Anura). Smithson. Contr. Zool., 457:1-99.

WASSERSUG, R.J. \& ROSENBERG, K. 1979. Surface anatomy of branchial food traps of tadpoles: A comparative study. J. Morphol. 159(3):393-425.

WEBER, L.N. \& CARAMASCHI, U. 2006. Descrição da morfologia oral interna de larvas do gênero Crossodactylus Duméril \& Bibron, 1841 (Amphibia, Anura, Leptodactylidae). Arq. Mus. Nac. Rio de Janeiro 64(2):141-149.

ZINA, J. \& HADDAD, C.F.B. 2005. Reproductive activity and vocalizations of Leptodactylus labyrinthicus (Anura: Leptodactylidae) in Southeastern Brazil. Biota Neotropica, 5(2): http://www.biotaneotropica.org.br/v5n2/ pt/abstract?article+BN00605022005.

Recebido em 10/09/07 Versão Reformulada recebida em 03/12/07 Publicado em 01/01/08 